\title{
Experimental Study on the Heat Transfer Performance of Porous Metal Fiber Sintered Sheet
}

\author{
Bin Liu ${ }^{1, a}$, Bo Ma ${ }^{1, b^{*}}$, Xin-gang Wang ${ }^{2, c}$, Li Zhang ${ }^{2, d}$, Jing-yang Ye $e^{1, e}$ \\ ${ }^{1}$ School of Mechanical and Automotive Engineering, South China University of Technology, \\ Guangzhou 510640, China \\ ${ }^{2}$ China Academy of Space Technology (Xi'an), Xi'an 710000, China \\ a liubinscut@163.com, ${ }^{\mathrm{b}}$ 380100411@qq.com, ${ }^{\mathrm{C}}$ dgang_2000@163.com, \\ dzhangli_casc@163.com ${ }^{\mathrm{e}}$ 406809573@qq.com
}

Keywords: Porous metal fiber sintered sheet, Heat transfer, porosity, Micro structure

Abstract. In this paper, a new kind of porous metal fiber sintered sheet (PMFSS) with high porosity was fabricated with cutting stainless steel fiber. And heat transfer experiments were conducted to investigate the effect of porosity and flow rate on the heat transfer properties of PMFSS. Metal fibers formed a three-dimensional reticulated structure and numerous connected micro pore structures in the PMFSS during the sintering process Test results showed that the heat transfer coefficient of the PMFSS increases with the increasing flow rate and $80 \%$ porosity sample shows the best performance. And the pressure drop increases with the decreasing porosity. Finally, comprehensive heat transfer coefficient of the material was investigated, and the $90 \%$ porosity shows the best performance.

\section{Introduction}

Nowadays, plate heat exchangers for its high efficiency and small size have been widely used in microelectronics cooling, aerospace, chemical engineering, biomedical field, robotics, telecommunications and automotive industries [1]. The most common structures used in the plate heat exchanger are micro channel [2] and porous media [3,4]. Porous media, for its high heat transfer capabilities, large specific area, light weight, has attracted more and more attention in recent years. The porous metal fiber sintered sheet (PMFSS), a new type of porous metals, has a three-dimensional reticulated structure featuring high porosity and large specific surface area. In the factual engineering applications, PMFSS has been widely used in filtration and separation [5], flow field and gas diffusion [6], catalytic reaction [7], and so on.

Numerous researchers have attempted to use porous media in heat exchangers. Hunt et al. [8] investigated the heat transfer performance of high porosity porous material. The study showed that the thermal performance of a metal-foam heat exchanger was superior to that of conventional finned-tube heat exchangers. Boomsma et al. [9] used open-cell metal foams as compact heat exchangers. It was shown that the open-cell metal foam heat exchangers gave rise to thermal resistances that are nearly one-third of the best available conventional heat exchanger. Furthermore, Lu et al. [10] presented the effects of various metal foam parameters on heat transfer. The results showed that the overall Nussle number of the metal foam filled pipe increased with the increase of relative density or pore density. Some researchers used analytical method to investigate the heat transfer performance of porous material. Zhao et al. [11] performed analytical studies of forced convection heat transfer characteristics of pipes filled with high-porosity, open-cell metal foam. It was shown that both the pore size and the porosity of metal foams played important roles in overall heat transfer performance. Another study was made by Bhattacharya et al. [12] where an analytical and experimental investigation for the determination of the effective thermal conductivity, permeability and inertial coefficient of high-porosity metal foams was presented. This work showed that the effective thermal conductivity of the foam depends strongly on the porosity and the ratio of the cross section of the fiber and the intersection. Finally, Lacroix et al. [13] presented an interesting work describing the pressure drop 
behavior of metal foam for varying values of porosity and apparent density through the use of an Ergun-type porosity model.

From the above reported work, it is found that the detailed study of the heat transfer performance of PMFSS is lacking in current literatures. This paper investigates the heat transfer performance of PMFSS produced by solid-state sintering of stainless steel fibers. The micro structure of PMFSS and the surface morphologies of the metal fibers are investigated. Finally, the effects of porosity and flow rate on the heat transfer performance of the PMFSS are analyzed.

\section{Experimental procedure}

Manufacturing process of PMFSS. As reported previously [14], the preparation of porous metal fiber sintered sheet mainly includes three processes, namely, the fabrication of cutting fibers, the mold-pressing of metal fibers, and the sintering with high temperature in a vacuum atmosphere.

First, long stainless steel fibers were fabricated by a multi-tooth tool on a horizontal lathe. The tool was installed on the turret with a setting angle of 45 degrees, and it had multiple tiny teeth fabricated through wire electrical discharge machining for achieving the simultaneous fabrication of multiple fibers. The parameters of the multi tooth tool used in this study are $m=0.3 \mathrm{~mm}, h=0.2 \mathrm{~mm}, \gamma_{o}=30^{\circ}$, and $\alpha_{o}=8^{\circ}$. Stainless steel $1 \mathrm{Cr} 18 \mathrm{Ni}$ Ti is used as the workpiece. The diameter of the cutting fibers was approximately $100 \mu \mathrm{m}$. The cutting fibers were collected by twining the fiber in a slab parallel to one another.

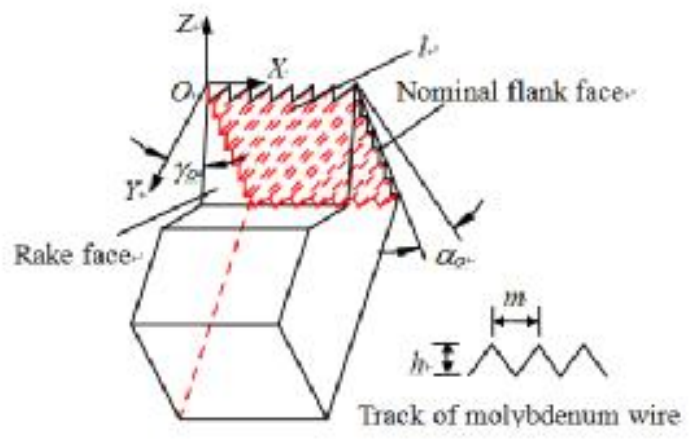

Fig.1. Schematic drawing of the multi-tooth tool

Second, the metal fibers were cut into segments with the length ranging from $10 \mathrm{~mm}$ to $20 \mathrm{~mm}$. Then, the metal fibers with random directions were uniformly put into the packing chamber of the mold pressing equipment and then pressure was applied by screwing the bolts. In this way, the semi-finished PMFSS with the same shape as the predetermined packing chamber was obtained. The dimension of the packing chamber in this study is $70 \mathrm{~mm}$ in length, $40 \mathrm{~mm}$ in width and $2 \mathrm{~mm}$ in height.

Later, the mold pressing equipment was put into a vacuum furnace to prevent the oxidation of metal fibers during sintering process. The sintering temperature and holding time were set as $1200^{\circ} \mathrm{C}$ and $60 \mathrm{~min}$, respectively. The heating rate is kept at $300^{\circ} \mathrm{C} / \mathrm{h}$ when the temperature is below $800^{\circ} \mathrm{C}$, while reduced to $200^{\circ} \mathrm{C} / \mathrm{h}$ as the temperature is above $800^{\circ} \mathrm{C}$. When the sintering process was completed, the furnace was cooled to room temperature. Finally, the mold pressing equipment was disassembled and the PMFSS with uniform thickness was formed.

The average porosity of the PMFSS can be calculated using the quality-volume method formulated by the following because the obtained porous metal has a regular geometric shape.

$$
\theta(\%)=\left(1-\frac{M}{\rho V}\right) \times 100
$$

where $V$ is the volume of the PMFSS $\left(\mathrm{cm}^{3}\right), M$ is the mass of the PMFSS $(\mathrm{g})$, and $\rho$ is the density of stainless steel $\left(\mathrm{g} / \mathrm{cm}^{3}\right)$. 
Testing system and procedures. In order to evaluate the heat transfer performance of the PMFSS, a small plate heat exchanger was designed in this study. The plate heat exchanger is made up of two cover plates and two cavities plates where two PMFSSs placed in. Some gaskets made of graphite were placed in the cover plates and the cavities plates to make sure the PMFSS contact well with the plate and prevent the water leak.

Fig. 2 shows the Schematic diagram of the plate heat exchanger test system, which consists of three main parts: hot water supply, cold water supply and data acquisition system. In the two water supply systems, the water temperature was controlled by two programmable temperature controllers. Water was used in the heat transfer test to simplify the experiment procedure.

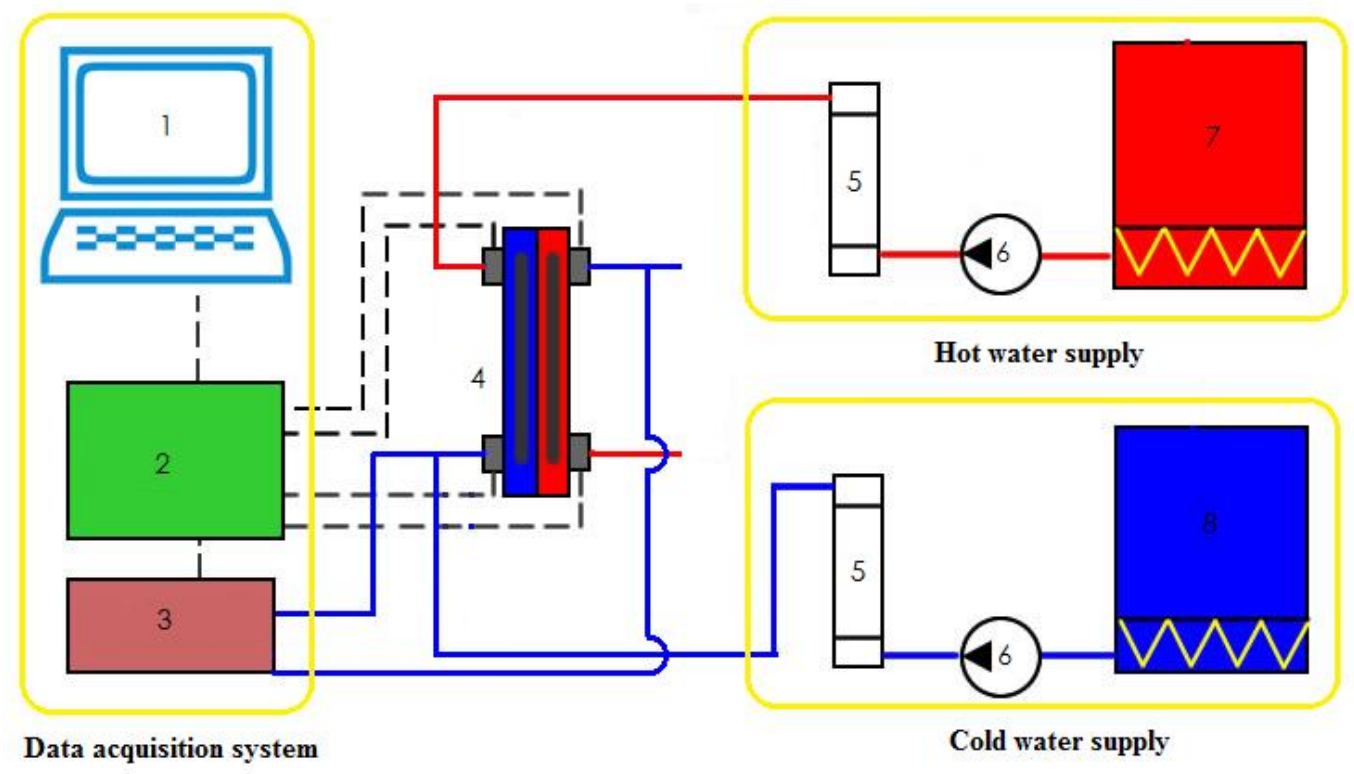

Fig.2. Schematic diagram of the plate heat exchanger test system.

(1) Computer, (2) Data acquisition instrument, (3) Capacitive pressure transmitter,

(4) Plate heat exchanger, (5) Flowmeter, (6) Pump, (7) Hot water tank, (8) Cold water tank

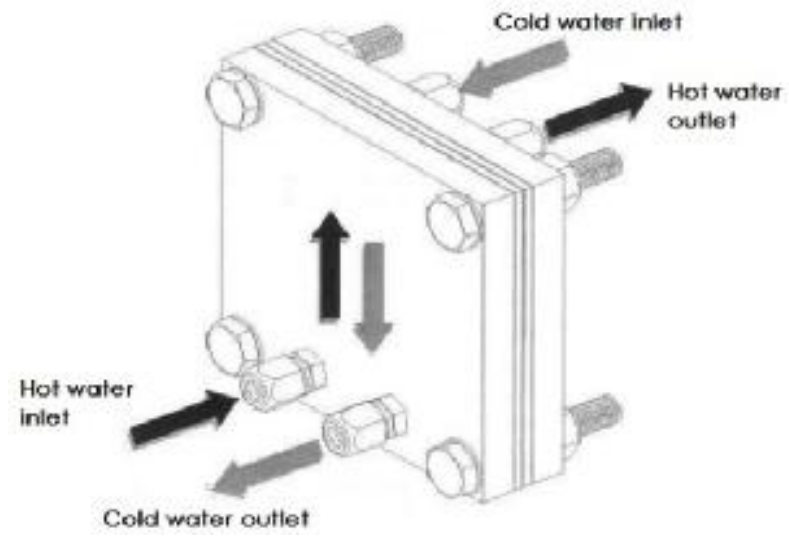

Fig.3. Flow direction of hot and cold water in the heat exchanger

In order to simulate the actual working condition of the plate heat exchanger, the water temperature at the hot inlet is kept at $85^{\circ} \mathrm{C}$, while the water temperature at the cold inlet is approximately a constant value $45^{\circ} \mathrm{C}$. Pumps were used to drive water to the system. The flow direction of the hot water and cold water were opposite to each other, as shown in fig 3.

During the experimental process, the flow rates of cold and hot water are controlled with two flowmeters separately, and the flow rates both increase from $2 \mathrm{~L} / \mathrm{h}$ to $10 \mathrm{~L} / \mathrm{h}$ by a step of $2 \mathrm{~L} / \mathrm{h}$. The water temperature is measured by k-type thermocouples, and then these data are transmitted to a computer via an Agilent-34970A data acquisition instrument. The pressure drops of the cold water were measured with a capacitive pressure transmitter. 
Moreover, the microscopic morphology of PMFSS was observed using a scanning electron microscopy (S-3700N, Hitachi, Japan).

Test evaluation. The heat transfer performance is estimated by the heat transfer coefficient per unit volume $\mathrm{K}_{\mathrm{V}}$, which is defined as follows:

$$
\left.K v=Q / \psi \bullet \Delta T_{m} \bullet V\right)
$$

where $V$ stands for the overall heat transfer volume. $\psi$ is the heat exchanger correction factor, here the value takes 1 .

$\mathrm{Q}$ is the total heat transfer quantity between the two fluids, which can be expressed by the heat absorbed by cold water.

$$
Q=\rho \cdot q_{\mathrm{m}} \cdot C_{p} \cdot\left(T_{\mathrm{ci}}-T_{\mathrm{co}}\right)
$$

where $q_{m}$ is the mass flow of cold water, and $C_{p}$ is the molar heat capacity of water at constant pressure

$\Delta T$ stands for the logarithm mean temperature difference as follows:

$$
\Delta T=\frac{\left(T_{\mathrm{hi}}-T_{\mathrm{co}}\right)-\left(T_{\mathrm{ho}}-T_{\mathrm{ci}}\right)}{\ln \frac{T_{\mathrm{hi}}-T_{\mathrm{co}}}{T_{\mathrm{ho}}-T_{\mathrm{ci}}}}
$$

Where $T_{h i}$ and $T_{h o}$ are the inlet and outlet temperatures of hot water, respectively. $T_{c i}$ and $T_{c o}$ are the inlet and outlet temperatures of cold water, respectively.

\section{Results and discussion}

Micro Structure of PMFSS. Fig. 4 shows the SEM images of the porous metal fiber sintered sheet sintered at $1200^{\circ} \mathrm{C}$ for $60 \mathrm{~min}$.

We can see from the figure that there are many sintering joints and connected pore structures in the PMFSS. Before sintering, stainless steel fibers were distributed randomly in the pressing mold. During the mold pressing process, metal fibers contact with each other and formed a porous structure. While, in the sintering process, the contact joints between metal fibers were easily to form the sintering joints under the effect of high sintering temperature and long holding time as a result of material migration. Finally, the sintering joints and the metal fibers formed the three-dimensional reticulated structure of the PMFSS, as shown in Fig. 4.
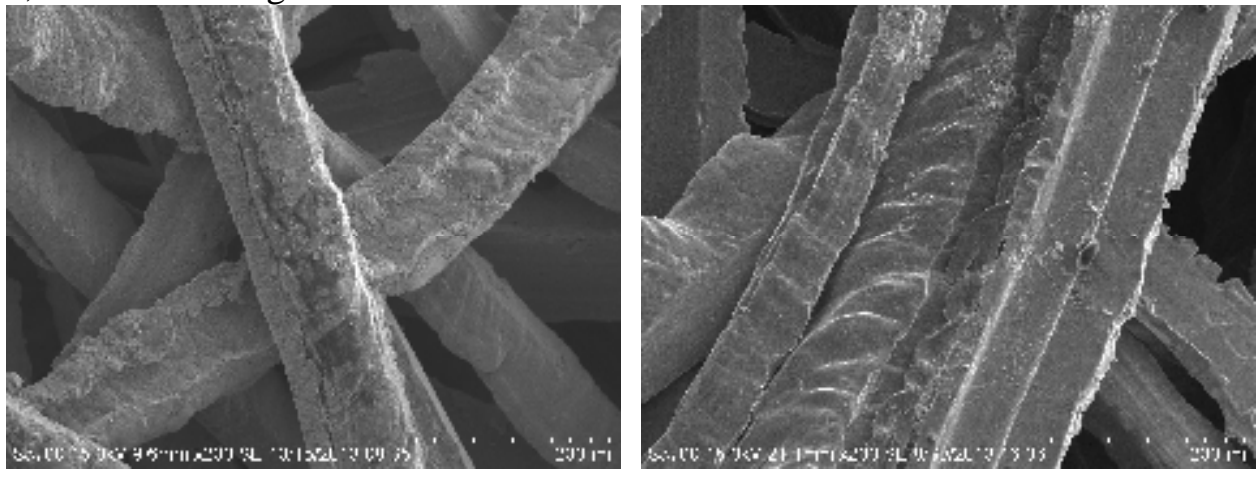

Fig.4. SEM images of porous metal fiber sintered sheet

(a) Point to point contact (b) fiber to fiber contact

As shown in the previous work [15], we could found that there were two kinds of sintering joints present in the PMFSS including point to point contact and fiber to fiber surface contact. And these two kinds of sintering joints formed two kinds of pore structures; they are point pore structure and long strip pore structure, as shown in Fig.4a and 4b, respectively. 
In addition, a lot of microstructures are found on the surface of the metal fibers in the PMFSS after sintering. These microstructures may not only increase the specific surface area, but also possibly improve the heat transfer performance of the PMFSS for that they can affect the flow condition of the liquid in the PMFSS.

Heat transfer coefficient per unit volume. Heat transfer coefficient is an important parameter to evaluate the heat transfer performance. Porosity, as the most important feature of porous materials, plays an important role in determining the properties of porous material. In this study, the effect of porosity on the heat transfer of PMFSS was investigated using three porosities of $70 \%, 80 \%$ and $90 \%$, and an empty experiment without any heat exchanger parts was done as a comparison.

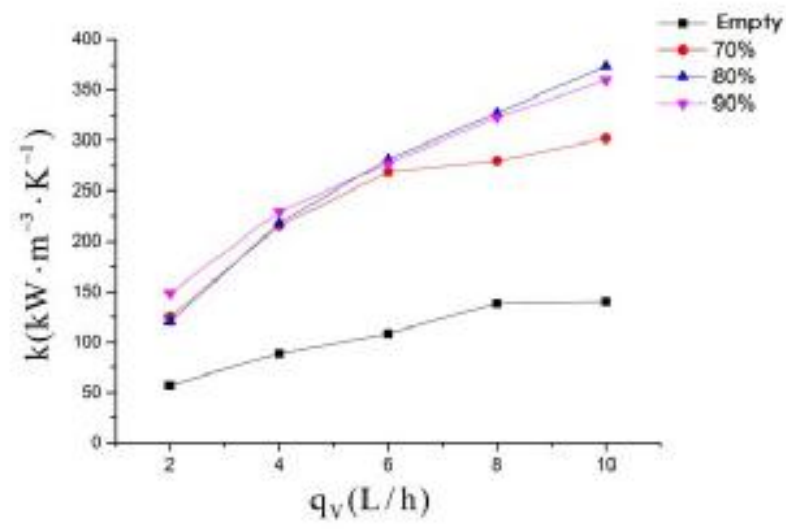

Fig. $5 \mathrm{Kv}$-qv curves of different porosities

Fig. 5 shows the heat transfer coefficient per unit volume of heat exchanger with different porosities sintered at $1200^{\circ} \mathrm{C}$ for $60 \mathrm{~min}$. We can see from the figure that the using of PMFSS can significantly increase the heat transfer coefficient of the heat exchanger and the porosity has significant influence on the heat transfer performance of PMFSS. And, it can be concluded from the figure that PMFSS with different porosities shows almost the same changing trend that the $\mathrm{K}_{\mathrm{v}}$ increases with the increase of flow rate. While the empty experiment changes only a little with the increasing flow rate.

When the $\mathrm{q}_{\mathrm{v}}$ is small between $2 \mathrm{~L} / \mathrm{h}$ and $6 \mathrm{~L} / \mathrm{h}$, the $\mathrm{K}_{\mathrm{v}}$ of PMFSSs with different porosities are almost the same. While, when the qv increases to $8 \mathrm{~L} / \mathrm{h}$ or $10 \mathrm{~L} / \mathrm{h}$, the $\mathrm{kv}$ of different samples differ greatly. At $10 \mathrm{~L} / \mathrm{h}$, the $\mathrm{K}_{\mathrm{v}}$ of $80 \%$ and $90 \%$ porosity samples are $360.2 \mathrm{kw} \cdot \mathrm{m}^{-3} \cdot \mathrm{K}^{-1}$ and $366.8 \mathrm{kw} \cdot \mathrm{m}^{-3} \cdot \mathrm{K}^{-1}$ respectively, while the $\mathrm{K}_{\mathrm{v}}$ of $70 \%$ porosity sample is only $298.6 \mathrm{kw} \cdot \mathrm{m}^{-3} \cdot \mathrm{K}^{-1}$. It can be indicated that high porosity porous metal fiber sintered sheet performs good in high flow rate.

The reasons are as follows: when flow rate is small, fluid flows slowly in the micro pores between metal fibers, so there is enough time for the heat exchange between hot water and cold water, and the samples with different porosities show almost the same properties. At this moment, the porosities have little effect on the heat transfer performance. While when the flow rate is higher, the fluid pass through of the porous metal is faster, the time for the heat exchange happens is not enough. But the samples with high porosities have more pore structures, and the heat transfer area is bigger than those with lower porosities. So the heat transfer performance of high porosity is better than that of low porosity at high flow rate.

These results can help us to design the PMFSS with higher heat transfer coefficient by improving the porosity of the PMFSS.

Pressure drop. Pressure drop is another parameter to evaluate the heat transfer performance. Three samples with $70 \%, 80 \%$ and $90 \%$ porosities were selected to investigate the pressure drop of the plate heat exchanger. The pressure drop between cold water inlet and outlet of each sample in the heat exchangers is shown in Fig.6. 


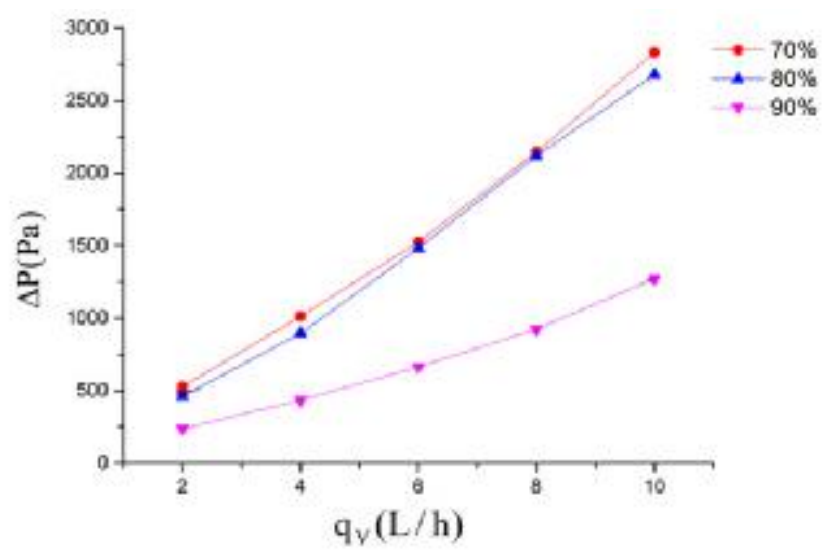

Fig.6 $\Delta$ P-qv curves of different porosities

We can see from the figure that the pressure drop increases with the flow rate, and the increasing trend is getting bigger with the flow rate. The pressure drop of $70 \%$ porosity is just $528.8 \mathrm{~Pa}$ in $2 \mathrm{~L} / \mathrm{h}$, while it reaches to $2828.1 \mathrm{~Pa}$ in $10 \mathrm{~L} / \mathrm{h}$.

And the pressure drop increases with the decreasing porosity especially in high flow rate. The biggest pressure drop happens in $70 \%$ porosity at the flow rate of $10 \mathrm{~L} / \mathrm{h}$, it is $2828.1 \mathrm{~Pa}$, and however, it is only 1280.3 of $90 \%$ porosity at $10 \mathrm{~L} / \mathrm{h}$. these results are similar with the results of Dukhan [16], where the pressure drop was significantly higher for low porosity metal foams.

These results are attributed to the following reasons. On the one hand, for the PMFSS with low porosity, it includes larger number of stainless steel fibers to form the three-dimensional reticulated structure, so there are not so many micro pores in the PMFSS. Furthermore, these structures reduce the effective volume for working fluid to pass through, lead to the increase of flow resistance. As flow resistance increased, the pressure drop increases. On the other hand, the average pore size of low porosity samples is smaller than those with high porosity; this will also lead to more resistance when water passes through the pore structure.

Therefore, these two aspects both increase the pressure drop of PMFSS with low porosity.

Comprehensive heat transfer coefficient. In practice, what we want is not only to enhance the heat transfer coefficient but also to decrease the pressure drop of the liquid in the heat exchanger, so a comprehensive parameter should be carried out to evaluate the comprehensive heat transfer performance. We choose the ratio of $\mathrm{K}_{\mathrm{v}}$ to $\Delta \mathrm{P}$ as the comprehensive heat transfer coefficient to estimate the heat transfer performance of PMFSS. Fig.7 shows the $\mathrm{K}_{\mathrm{v}} / \Delta \mathrm{P}$-qv curves of different porosities in the heat exchanger.

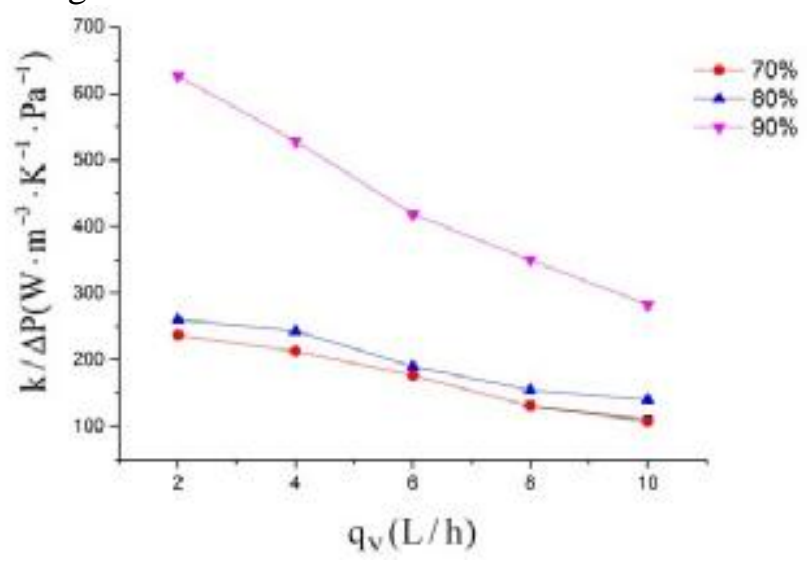

Fig. $7 K_{\mathrm{v}} / \Delta p-q_{\mathrm{v}}$ curves of different porosities

It can be concluded from the figure that the $\mathrm{K}_{\mathrm{v}} / \Delta \mathrm{P}$-qv curves show the similar changing trend during the experimental range, that is it decreases with the increase of flow rate and decreases with decreasing porosity. 
The best comprehensive heat transfer coefficient of the three samples all were obtained at the flow rate of $2 \mathrm{~L} / \mathrm{h}$, the value reaches to $628.3 \mathrm{~W} \cdot \mathrm{m}^{-3} \cdot \mathrm{K}^{-1} \cdot \mathrm{Pa}^{-1}$ of the $90 \%$ porosity, while the value of $70 \%$ and $80 \%$ porosities are only $236.9 \mathrm{~W} \cdot \mathrm{m}^{-3} \cdot \mathrm{K}^{-1} \cdot \mathrm{Pa}^{-1}$ and $258.6 \mathrm{~W} \cdot \mathrm{m}^{-3} \cdot \mathrm{K}^{-1} \cdot \mathrm{Pa}^{-1}$ respectively.

From these results, we can see that one way to increase the heat transfer coefficient per unit volume of a conventional plate heat exchanger is to replace the fins by a porous structure. Under this condition, the heat exchange surface area can reach very high values. However, this increase amplifies the pressure drop of the fluid circulating in the porous matrix. It is therefore necessary to determine the optimal parameters of the porous medium in order to maximize the heat transfer with regard to the pressure drop. These results above can give us some useful information in practice. In order to get the best heat transfer performance, we could choose different porosity of PMFSS, and adapt the heat transfer parameters to get the optimum result.

\section{Conclusion}

The following conclusion can be concluded from this work,

(1)A novel porous metal fiber sintered sheet (PMFSS) was fabricated via solid sintering process with cutting stainless steel fiber, the manufacturing process of the PMFSS can be divided into three steps: the fabrication of cutting fibers, mold pressing process and sintering process. This material has a three-dimensional reticulated structure featuring high porosity and large specific surface area. There are numerous micro structures on the surface of the metal fiber and the PMFSS.

(2)PMFSS can enhance the heat exchange performance obviously. Porosity and flow rate have important effect on the heat transfer performance of the PMFSS. Experimental result shows that the heat transfer coefficient increases with the flow rate and the $80 \%$ porosity sample shows the best performance. And the pressure drop increases with the flow rate and the decreasing porosity. Finally, the comprehensive heat transfer coefficient decreases with the decreases of flow rate and the decreasing porosity. The best value was obtained at $90 \%$ porosity at $2 \mathrm{~L} / \mathrm{h}$, it is $628.3 \mathrm{~W} \cdot \mathrm{m}^{-3} \cdot \mathrm{K}^{-1} \cdot \mathrm{Pa}^{-1}$.

\section{Acknowledges}

This work was supported by the National Nature Science Foundations of China (No. 51275180, 51475172, 51375176).

\section{References}

[1] A.E. Bergles. Heat transfer enhancement the encouragement and accommodation of high heat fluxes [J]. ASME Journal of Heat Transfer, 1997, 119(1):8-19

[2] P.X. Jiang, M.H. Fan, G.S. Si, et al. Thermal-hydraulic performance of small scale

micro-channel and porous-media heat-exchangers[J]. International Journal of Heat and Mass Transfer, 2001, 44(5): 1039-1051

[3] J. Banhart. Manufacture, characterisation and application of cellular metals and metal foams. Progress in Materials Science, 2001, 46(6) 559-632

[4] A.S. Abduljalil, Z.B. Yu, A.J. Jaworski, Selection and experimental evaluation of low-cost porous materials for regenerator applications in thermoacoustic engines [J] Materials \& Design,2011,32 (1):217-228

[5] A. Nabovati, E.W. Llewellin, A.C.M. Sousa, A general model for the permeability of fibrous porous media based on fluid flow simulations using the lattice Boltzmann method[J] Composites Part A: Applied Science and Manufacturing, 2009, 40(6-7): 860-869.

[6] J.K. Kuo, C.K. Chen, A novel Nylon-6-S316L fiber compound material for injection molded PEM fuel cell bipolar plates [J]. Journal of Power Sources,2006, 162(1):207-214 
[7] Y Tang, W Zhou, M.Q. Pan, H.Q. Chen, W.Y. Liu, H. Yu. Porous copper fiber sintered felts: an innovative catalyst support of methanol steam reformer for hydrogen production [J]. International Journal of Hydrogen Energy, 2008, 33(12): 2950-2956.

[8] M.L.hunta, C.L.Tiena. Effects of thermal dispersion on forced convection in fibrous media [J]. International Journal of Heat and Mass Transfer, 1988, 31(2):301-309

[9] K. Boomsma, D. Poulikakos, F. Zwick, Metal foams as compact high performance heat exchangers [J], Mechanics of Materials, 2003,35 (12) :1161-1176.

[10] W. Lu, C.Y. Zhao, S.A. Tassou, Thermal analysis on metal foam filled heat exchangers. Part I: Metal foam filled pipes[J], International Journal of Heat and Mass Transfer,2006,49(15-16): 2751-2761.

[11] C. Y. Zhao, W. Lu and S. A. Tassou, Thermal analysis on metal-foam filled heat exchangers, Part II: tube heat exchangers [J], International Journal of Heat and Mass Transfer,2006,49(15-16): 2762-2770.

[12] A. Bhattacharya, V.V. Calmidi, R.L. Mahajan, Thermophysical properties of high-porosity metal foams [J], International Journal of Heat and Mass Transfer ,2002,45:1017-1031.

[13] M. Lacroix, P. Nguyen, D. Schweich, C.P. Huu, S. Savin-Poncet, D. Edouard, Pressure drop measurements and modeling on $\mathrm{SiC}$ foams $[\mathrm{J}]$, Chemical Engineering Science, 2007,62:3259-3267.

[14] C.B. Fang, Z.P. Wan, B Liu, L.S. Lu. A Novel Sintered Stainless Steel Fiber Felt with Rough Surface Morphologies [J]. Advances in Materials Science and Engineering,2014:1-7

[15] Y Tang, W Zhou, J.H. Xiang, W.Y. Liu, M.Q. Pan. An innovative fabrication process of porous metal fiber sintered felts with three-dimensional reticulated structure $[\mathrm{J}]$. Mater Manuf Process 2010;25:565-71.

[16] N. Dukhan, Correlations for the pressure drop for flow through metal foam[J], Experiments in Fluids, 2006, 41(4): 665-672 